\title{
Comparing ERAS-outpatient
} versus standard-inpatient hip and knee replacements: a mixed methods study exploring the experience of patients who underwent both

\author{
Alexandre Hardy 1,2,3, Jonathan Gervais-Hupé ${ }^{4,5,6}$, François Desmeules ${ }^{3,4}$, Anne Hudon 4,5,6, \\ Kadija Perreault ${ }^{7,8}$ and Pascal-André Vendittoli ${ }^{2,3,9,10^{*}}$
}

\begin{abstract}
Background: Optimizing patients' total hip and knee arthroplasty (THA/TKA) experience is as crucial for providing high quality care as improving safety and clinical effectiveness. Yet, little evidence is available on patient experience in standard-inpatient and enhanced recovery after surgery (ERAS)-outpatient programs. Therefore, this study aimed to gain a more in-depth understanding of the patient experience of ERAS-outpatient programs in comparison to standard-inpatient programs.
\end{abstract}

Methods: We conducted a convergent mixed methods study of 48 consecutive patients who experienced both standard-inpatient and ERAS-outpatient THA/TKA contralaterally. A reflective thematic analysis was conducted based on data collected via a questionnaire. Bivariate correlations between the patient experience and patients' characteristics, clinical outcomes and care components satisfaction were performed. Then, the quantitative and qualitative data were integrated together.

Results: The theme Support makes the difference for better and for worse was identified by patients as crucial to their experience in both joint replacement programs. On the other hand, patients identified 3 themes distinguishing their ERAS-outpatient from their standard-inpatient experience: 1) Minimizing inconvenience, 2) Home sweet home and 3) Returning to normal function and activities. Potential optimization expressed by patients were to receive more preoperative information, additional postoperative rehabilitation sessions, and ensuring better coherence of care between hospital and home care teams. Weak to moderate positive and statistically significant correlations were found between patients'THA/TKA experience and satisfaction with pain management, hospital stay, postoperative recovery, home care, and overall results $\left(r_{s}=+[0.36-0.66], p\right.$-value $\left.<0.01\right)$.

Conclusion: Whatever the perioperative program, the key to improving patients'THA/TKA experience lies in improving support throughout the care episode. However, compared to standard-inpatient care, the ERAS-outpatient program improves patients' experience by providing dedicated support in postoperative care, reducing postoperative inconvenience, optimizing pain management, returning home sooner, and recovering and regaining function sooner.

*Correspondence: pa.vendittoli@me.com

${ }^{2}$ Hôpital Maisonneuve-Rosemont, Surgery Department, Université de Montréal, Montreal, Quebec, Canada

Full list of author information is available at the end of the article

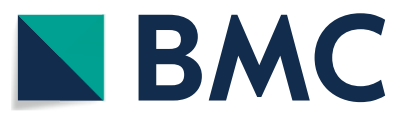

(c) The Author(s) 2021. Open Access This article is licensed under a Creative Commons Attribution 4.0 International License, which permits use, sharing, adaptation, distribution and reproduction in any medium or format, as long as you give appropriate credit to the original author(s) and the source, provide a link to the Creative Commons licence, and indicate if changes were made. The images or other third party material in this article are included in the article's Creative Commons licence, unless indicated otherwise in a credit line to the material. If material is not included in the article's Creative Commons licence and your intended use is not permitted by statutory regulation or exceeds the permitted use, you will need to obtain permission directly from the copyright holder. To view a copy of this licence, visit http://creativecommons.org/licenses/by/4.0/. The Creative Commons Public Domain Dedication waiver (http://creativeco mmons.org/publicdomain/zero/1.0/) applies to the data made available in this article, unless otherwise stated in a credit line to the data. 
Patients'THA/TKA experience could further be enhanced by optimizing the information provided to the patient, the rehabilitation program and the coherence between care teams.

Keywords: Arthroplasty, replacement, knee, Arthroplasty, replacement, hip, Enhanced recovery after surgery, Fasttrack, Outpatient, Patient experience, Patient outcome assessment, Mixed methods research

\section{Introduction}

Fast-track protocols represent a potential solution to the challenges posed by the expected increase in demand for total hip and knee arthroplasty (THA/TKA) over the coming decades and wait times exacerbated by the COVID pandemic [1-3]. The shift from inpatient to outpatient programs for THA/TKA is also attractive due to increased capacity and bed availability, as well as reduced length of stay and health care costs [4-6]. However, the main objective for transitioning to an ambulatory practice should be to improve the recovery process to a level where it is safe for the patients to return home on the day of surgery [7]. To achieve this objective, outpatient programs should be based on enhanced recovery after surgery (ERAS) principles that focus on optimizing all aspects of perioperative care to ensure effective, secure and rapid recovery $[8,9]$. To offer high-quality care, ERAS-outpatient THA/TKA aim to optimize the three pillars of quality of care as described by the National Health Service: patient safety, effectiveness of care and patient experience $[10,11]$.

A recent study by Hardy \& al [12]. compared a THA/ TKA ERAS-outpatient to standard-inpatient care on all three pillars and found that the ERAS-outpatient reduced complications and opioid consumption, enabled faster functional recovery and improved patients' satisfaction and experience on a visual analog scale. Other studies corroborated these findings but yet, very little evidence is available on patient experience [13-17]. Patient experience is a complex concept, representing "the sum of all interactions, shaped by an organization's culture, that influence patient perceptions, across the continuum of care" and that understanding the patient experience or factors enhancing it, goes beyond quantitative assessment alone $[18,19]$. Therefore, this study's overarching objective was to gain a more in-depth understanding of the patient experience for both STD-inpatient and ERASoutpatient THA/TKA by combining the strengths of quantitative and qualitative approaches [20]. Specifically, in subjects who experienced both an ERAS-outpatient and a STD-inpatient program, we wanted to compare their experience and identify elements that could be optimized. We also sought to determine whether and how patient characteristics, clinical outcomes and satisfaction of care components are associated with patients' experience.

\section{Methods}

Study design, patient selection and characteristics

This study used a convergent mixed methods single subject design (Quan + Qual) to gain a more in-depth understanding of patient experience for both STD-inpatient and ERAS-outpatient THA/TKA. In October 2020, in conjunction with Hardy \& al. study [12], we recruited the first 50 adult patients who sequentially underwent both programs for the same primary surgery on contralateral articulations in a Canadian tertiary hospital and who were able to communicate in French or English. Out of all eligible participants, 48 gave an informed written consent and enrolled in the study; two patients declined because they were not interested in participating. The criteria patients had to fit to undergo the ERAS-outpatient program were described by Vendittoli et al. [13] The study was conducted in accordance with the Declaration of Helsinki and this study was approved by our Institutional Review Board (Comité déthique de la recherche du CIUSSS de l'Est-de-l'Île-de-Montréal \#2021-2420). Full details of participants' characteristics were presented in a previous paper [12] and are summarized in Table 1.

\section{STD-inpatient and ERAS-outpatient programs description}

In both programs, patients met with several health professionals weeks before the day of surgery and an additional informative group session was offered in the ERAS-outpatient program. Recommendations before surgery were the same for both programs except that fasting after midnight was required for STD-inpatient, whereas they could drink clear liquids up to $2 \mathrm{~h}$ before the ERAS-outpatient operation. Perioperative care provided in the STD-inpatient program varied according to the patient's characteristics and surgeon's preferences, whereas it was standardized for ERAS-outpatient (every patient received the same interventions). Surgical approach and implants were identical for both programs. The first physiotherapy session aimed to be performed on postoperative day 1 for STD-inpatient and in the first 4-6h after surgery for ERAS-outpatient. STD-inpatient patients were expected to be hospitalized 1-3 days for a THA and 3-5 days for a TKA whereas ERAS-outpatient cases were expected to be discharged the same day of surgery (THA) or in $<24 \mathrm{~h}$ (TKA). Regardless of the program, all patients returned home after discharge, followed the same rehabilitation program, and received 
Table 1 Patients' Characteristics

\begin{tabular}{|c|c|c|c|}
\hline & ERAS-outpatient & STD-Inpatient & $P$-value \\
\hline Number of participants & 48 & & \\
\hline \multicolumn{4}{|l|}{ Gender } \\
\hline (Men:Women) & 27 (56.2\%): $21(43.8 \%)$ & & \\
\hline Average Age at Surgery & $60.0(45.0-76.0,7.8)$ & $52.9(33.0-70.0,8.3)$ & $<0.001^{\mathrm{a}}$ \\
\hline Average Body Mass Index & $28.1(20.7-37.6,4.1)$ & $28.3(19.3-35.0,4.0)$ & $0.638^{\mathrm{a}}$ \\
\hline ASA Physical Status & & & $0.007^{b}$ \\
\hline 1 & $17(35.4 \%)$ & $25(52.1 \%)$ & \\
\hline 2 & $30(62.5 \%)$ & $23(47.9 \%)$ & \\
\hline 3 & $1(2.1 \%)$ & 0 & \\
\hline \multicolumn{4}{|l|}{ Intervention } \\
\hline THA & $36(75.0 \%)$ & & \\
\hline TKA & $12(25.0 \%)$ & & \\
\hline
\end{tabular}

a Paired T-test

${ }^{\mathrm{b}}$ Marginal homogeneity test

similar home care services. STD-inpatient surgeries took place between 2000 and 2018 and were all performed before ERAS-outpatient surgeries, performed between 2017 and 2020. Detailed description of both ERAS-outpatient and STD-inpatient programs were previously presented by Vendittoli et al. [13] and Hardy et al. [12]

\section{Data collection}

Patient characteristics and postoperative data: pain on a numeric rating scale (NRS) [0-10], opioid consumption in milligram morphine equivalent, numbers of complications measured with the Clavien-Dindo classification, complications' morbidity rated with the Comprehensive Complication Index, numbers of unplanned episodes of care, length of stay in hours, and time in days to reach functional recovery landmarks at the hospital were collected retrospectively from medical records. Through the Patient Experience questionnaire [Supplementary Material: Appendix A], we collected prospectively, at the last follow-up ( 9 months to several years after surgeries): the time to reach functional recovery landmarks at home, patient overall care experience, and patient satisfaction of care components (preparation before surgery, hospital stay, home care, pain management, postoperative recovery, wound care, overall result) on visual analog scale (VAS) [0-100]. When patient overall care experience and patient satisfaction of care components on VAS differed between programs, patients were asked open-ended questions to provide a deeper understanding of their experience. In addition, participants were invited to explain their answers when questioned about which program they would recommend. Moreover, they were invited to suggest any improvement they thought that could be made to the programs to enhance their experience. To prevent any potential wording bias related to the names of the programs "Enhanced Recovery after Surgery" versus "Standard", all questions referred to "Right" and "Left" sides instead. Once the survey completed, answers were categorized to the appropriate program (ERAS-outpatient or STD-inpatient) before proceeding to analyses. All the data collected were deidentified and stored in a secured REDCap database [Research Electronic Data Capture, Vanderbilt University, TN, USA].

\section{Data processing and analysis Qualitative data}

All text data gathered through open-ended questions in the Patient Experience questionnaire were qualitatively analyzed in QDA Miner [QDA Miner, Version 5.0, Provalis Research, Montreal, QC, CAN] using a reflexive thematic analysis inspired by Braun \& Clark [21]. First, two authors (A. Hardy and J.G.H.) read the entire data set many times to become familiar with it. They recorded their thoughts in a research audit trail. Second, based on an inductive and semantic approach, both investigators generated initial codes independently for five participants. Then, they compared their initial codes, made modifications if needed, and elaborated a common codebook based on their consensus. Third, they repeated this process for five more participants to assure trustworthiness regarding the analytical approach. Fourth, A. Hardy coded the data of remaining participants, and afterwards grouped codes into broader categories and developed overarching themes. Fifth, he created a thematic map to visualize the relationships between themes and to sort main themes from secondary ones. Sixth, the representation of results was debriefed and discussed with all 
investigators which led A. Hardy to reread the entire data set and refined themes to form a coherent whole that truly captured the essence of the participants' experiences. When refinements stopped producing significant improvements, final themes were approved by the whole research team.

\section{Quantitative data}

Relations between patient overall care experience on the VAS [0-100] and continuous data on patient characteristics, clinical outcomes and patient satisfaction of care components were analyzed with Pearson or Spearman bivariate correlations. Point-biserial correlations were realized when patient characteristics and clinical outcomes data were dichotomous. A two-sided alpha level of significance of 0.05 was used and all tests were performed with SPSS - 25 [IBM SPSS Statistics for Macintosh, Version 25.0. IBM Corp Armonk, NY, USA].

\section{Quantitative and qualitative data integration}

The qualitative and quantitative data were first analyzed separately. Then, they were integrated together in the discussion and compared to the literature to provide a more in-depth understanding of patient experience for both STD-inpatient and ERAS-outpatient THA/TKA [22].

\section{Results}

\section{Qualitative results}

The qualitative analysis allowed to identify themes that provided a better understanding of the patient experience during THA/TKA. One theme was commonly present among both programs: Support makes the difference for better and for worse. Three other themes captured improvements in the patient experience due to the ERAS-outpatient program compared to STD-inpatient one: 1) Minimizing inconvenience, 2) Home sweet home and 3) Returning to normal function and activities. Finally, another theme captured improvements that could be made to the patient experience: Room for improvement. Quotes from Francophone participants were translated into English by a native English speaker.

\section{Support makes the difference for better and for worse}

No matter the phase of care and the program, participants spontaneously evoked their appreciation for benefiting from a well-framed structure from beginning to end. They felt better supported and confident when healthcare providers identified themselves and answered their questions. They appreciated healthcare providers who are good listeners and valued receiving clear information, so they were not left in the unknown.
"For me, everything was perfect, the information was clear, the care from start to finish, everything was well managed, I never felt in the unknown." (Patient 40).

On the other hand, they were dissatisfied when the first contact with the healthcare team was judged as non-professional or when their surgeon did not visit them after the intervention. Participants also discussed how the support provided on the ward after surgery affected their experience. Some disliked their STD-inpatient experience because they thought that postoperative care was disorganized and of poor quality. They observed that the staff seemed overwhelmed and felt that they did not care about them as several patients overheard the staff arguing about who will assume responsibility for their care. Others shared how they felt let down because they experienced complications and healthcare providers took a long time to acknowledge and address them. In contrast, other participants appreciated the competency and professionalism of healthcare workers dedicated to the outpatient ward and some even expressed they felt pampered. Participants also discussed the support they received at home after surgeries. Most of them enjoyed the home care services provided by nurses and physiotherapists in both programs. They said they appreciated the professionalism of home care professionals as they took all the time needed to provide care, answer questions and to refer to other healthcare professionals when required. Some participants even said that having a dedicated member of the orthopaedic team made available to answer their questions and tamper their fears and anxieties was a huge plus. On the other hand, a few participants had mixed feelings about the quality of care they received. These participants considered that the healthcare workers who came to assist them were incompetent and unprofessional. They stated that home care providers changed personnel often, were not familiar with their file, did not follow the instructions given by the surgical team, did not detect complications, were unable to answer their inquiries and sometimes did not show up to provide the prescribed care. Overall, patients expressed the importance of support from the healthcare team throughout the entire care process in both programs. However, when participants reported differences between programs, they usually felt better supported in the ERAS-outpatient program because they benefited from workers dedicated to this program in the ward.

"Left hip, day surgery protocol. AWESOME! Qualified and dedicated staff for day surgeries. Always someone close by to make sure everything goes well. I had questions about my medication, the orthopaedic nurse referred me to a pharmacist right away and I quickly got the right advice. I felt like I was being treated like a princess because the people were so attentive." (Patient 34). 


\section{Minimizing inconvenience}

Patients noted differences between programs regarding prevention and management of adverse events. For pain management, participants preferred the ERAS-outpatient program because their pain was better relieved with less medication and being home sooner allowed them to take analgesics as prescribed without having to wait for the hospital staff as during STD-inpatient. Patients also liked the opioid-sparing analgesic modalities of the ERAS-outpatient because it prevented side effects associated with opioids, including vomiting or constipation, and alleviated their fear of developing an addiction. Moreover, patients noted that staples used to close the surgical wound were replaced by sutures and tissue adhesive in the ERAS-outpatient program which eliminated their bothersome side effects like wound discharge and itching in addition to the inconvenience and pain of having staples removed. Many participants also preferred the use of tissue adhesive (ERAS-outpatient) because it enabled them to shower sooner and they felt that their wounds healed better and more quickly, resulting in smaller and more aesthetical scar.

"With the glue, it's fantastic, no discharge, you can shower faster, the scar heals faster and looks better" (Patient 40).

Overall, patients reported the type of anesthesia as being a major difference between the two programs. Some stated that, after ERAS-outpatient, they did not suffer from prolonged motor blockade nor from urinary retention unlike during STD-inpatient. Others reported that the epidural-sedation anesthesia used in ERASoutpatient was as efficient as the spinal anesthesia used in STD-inpatient, but better tolerated with fewer side effects. They added that feeling less sedated enabled waking up and recovering more quickly after the surgery.

"Easier to wake up from surgery (ERAS-outpatient) and go home the next day, less sick. I was much better in general when I came out of the operating room than at the first operation (STD-inpatient)." (Patient 10).

Globally, participants expressed that postoperative inconveniences were better prevented or managed with ERAS-outpatient.

\section{Home sweet home}

For some patients, the expectation to go home only hours after surgery was at first a source of anxiety. Nevertheless, they were reassured because a caregiver would be with them. Because they felt the ward can be a harsh setting in which to recover, most patients appreciated the reduced hospital length of stay with ERAS-outpatient.

"The less time spent in hospital the better! Long live day surgery!" (Patient 45).
They evoked the difficulty of getting a good night of sleep because of the noise, their lack of interest in the hospital food and the anxiety-inducing atmosphere of the environment. Even when they reported excellent care at the hospital for their first surgery, patients appreciated leaving the hospital as early as possible and found convalescence was easier and better for the mind at home.

"Despite the good care of the nursing staff, recovery is easier at home, in my environment, much easier and better for the spirit." (Patient 25).

\section{Returning to Normal function and activities}

Most participants reported major differences between programs in the time and effort required to return to normal function. With ERAS-outpatient, they expressed their delight in performing activities of daily living, such as walking and climbing stairs on the day of surgery, which was not the case after STD-inpatient. Some even shared their astonishment in being able to go shopping and going back to work only a couple of days later.

"For the left leg with the day surgery, the recovery was really superfast. 3 days later I was at the grocery shop and the next day I was going to the office! Much faster than the first operation which still went very well in terms of recovery." (Patient 45).

Participants noted that the overall recovery process was generally quicker and easier following the ERASoutpatient THA/TKA because of what they perceived as enhanced supervision and better adapted exercises. They noted that fewer physiotherapy sessions were needed to recover compared to STD-inpatient.

"For the right side (STD-inpatient) much more time in physio 1-2 months, whereas the left side (ERAS-outpatient) 3 visits in physio and finished thereafter." (Patient 3).

However, in both programs, some patients experienced obstacles such as pain and deficits in strength and flexibility that hampered their functional recovery. For very few patients, these difficulties persisted and negatively tainted their experience, whereas most patients were happy with the overall results of both THA/TKA.

\section{Room for improvement}

Improvements to THA/TKA programs were highlighted by some patients. For the preparation phase, patients suggested to present patients' testimonies of previous experiences and to further explain the patient's role in the process to optimize recovery. They further recommended that surgeons come visit their patients in the recovery room. Moreover, patients suggested that having more physiotherapy sessions, as needed, would be beneficial.

"Having more physiotherapy at home if needed: I believe this is the key after a successful surgery" (Patient 8). 
Table 2 Relations Between Patient Overall Care Experience and Patients' Characteristics and Clinical Outcomes

\begin{tabular}{|c|c|c|c|c|}
\hline \multirow[t]{2}{*}{ Patient characteristics } & \multicolumn{2}{|c|}{ ERAS-Outpatient } & \multicolumn{2}{|c|}{ STD-Inpatient } \\
\hline & $\begin{array}{l}\text { Spearman } \\
\text { correlation } \\
\text { coefficient }\end{array}$ & $P$-value & $\begin{array}{l}\text { Spearman } \\
\text { correlation } \\
\text { coefficient }\end{array}$ & $P$-value \\
\hline Gender & .044 & 0.771 & .137 & 0.359 \\
\hline Age at surgery & .148 & 0.321 & .158 & 0.288 \\
\hline Body Mass Index & .152 & 0.309 & .136 & 0.368 \\
\hline ASA Physical Status & .163 & 0.273 & .089 & 0.553 \\
\hline Intervention (THA/TKA) & .091 & 0.545 & -.188 & 0.205 \\
\hline \multicolumn{5}{|l|}{ Postoperative variables } \\
\hline Pain in the recovery room (NRS 0-10) & .002 & 0.99 & -.071 & 0.651 \\
\hline Pain in the ward, surgery day (NRS 0-10) & .235 & 0.112 & -.043 & 0.779 \\
\hline $\begin{array}{l}\text { Mean Opioid Consumption in Morphine Milligram Equivalents in the First } \\
8 \text { Hours After Surgery }\end{array}$ & -.163 & 0.275 & -.087 & 0.56 \\
\hline Complications: All Grades & .060 & 0.689 & .020 & 0.892 \\
\hline Complications: Grade 1 & .053 & 0.721 & .085 & 0.572 \\
\hline Complications: Grade 2 & -.037 & 0.806 & -.273 & 0.063 \\
\hline Comprehensive Complication Index & -.023 & 0.876 & -.163 & 0.273 \\
\hline Emergency Care Unit visits without intervention & -.239 & 0.106 & -.039 & 0.794 \\
\hline Emergency Care Unit visits with interventions & -.015 & 0.922 & .022 & 0.882 \\
\hline Clinic consultations without intervention & -.057 & 0.706 & .034 & 0.822 \\
\hline Clinic consultations with interventions & & & -.257 & 0.082 \\
\hline First rise/standing (in days) & .232 & 0.117 & .192 & 0.218 \\
\hline Walking (in days) & .058 & 0.71 & .144 & 0.382 \\
\hline Going up and down the stairs (in days) & .205 & 0.181 & -.003 & 0.985 \\
\hline Length of stay (in hours) & .095 & 0.527 & -.162 & 0.281 \\
\hline First shower (in days) & -.103 & 0.497 & -.245 & 0.104 \\
\hline Walking without technical aid (in days) & -.005 & 0.972 & -.106 & 0.484 \\
\hline Going up and down the stairs without technical aid (in days) & -.014 & 0.923 & -.031 & 0.836 \\
\hline ADLs: dressing, toileting, walking indoor alone, etc. (in days) & -.19 & 0.201 & -.174 & 0.248 \\
\hline iADLs: cooking, cleaning, shopping, etc. (in days) & -.007 & 0.965 & -.109 & 0.472 \\
\hline Mild physical activities: cycling, swimming, walking, etc. (in weeks) & -.085 & 0.584 & .088 & 0.567 \\
\hline Intense physical activities: running, playing tennis, skiing, etc. (in weeks) & .28 & 0.166 & .106 & 0.59 \\
\hline Return to light-duty work (in weeks) & .285 & 0.121 & .097 & 0.562 \\
\hline Return to regular work without limitation (in weeks) & .193 & 0.344 & .069 & 0.707 \\
\hline
\end{tabular}

Also, participants recommended that communication between the hospital team and home care teams be improved to convey more coherent instructions.

"Improved communication between the hospital physio and the $C L S C^{1}$ physio. The hospital physio explained to me that with the new protocol (ERAS-outpatient) I could start walking, climbing stairs and even riding a stationary bike faster whereas the CLSC asked me to wait." (Patient 1).

Finally, they advocated to maintain having a dedicated resource person from the hospital team easily reachable by phone to answer inquiries.

\footnotetext{
${ }^{1}$ Centres locaux de services communautaires (CLSC) are the home care providers.
}

\section{Quantitative results}

The bivariate correlation between patients' overall care experience and patient characteristics and clinical outcomes showed only poor association $r_{s}= \pm[.000-0.299]$ that was not statistically significant $(p>0.05$; Table 2$)$. The positive associations between patients' overall care experience and satisfaction of care components in the ERASoutpatient were found to be fair $r_{s}=+[0.400-0.599]$ to moderate $r_{s}+[0.600-0.799]$ and were all considered statistically significant $(p<0.01$; Table 3$)$. The spearman correlation coefficients between these same variables in the STD-inpatient showed generally weaker positive associations, ranging from poor and non-statistically significant $(\mathrm{p} \geq 0.108)$ for preparation and wound care to fair and 
Table 3 Relations Between Patient Overall Care Experience and Satisfaction of Care Components (VAS 0-100)

\begin{tabular}{|c|c|c|c|c|c|c|c|}
\hline \multirow[t]{2}{*}{ Variables } & \multicolumn{3}{|c|}{$\begin{array}{l}\text { Patient Overall Care Experience and } \\
\text { Satisfaction of care components (VAS 0-100) } \\
\text { [12] }\end{array}$} & \multicolumn{2}{|c|}{ ERAS-outpatient } & \multicolumn{2}{|c|}{ STD-inpatient } \\
\hline & $\begin{array}{l}\text { ERAS-outpatient } \\
\text { Mean } \\
\text { (Min-Max, SD) }\end{array}$ & $\begin{array}{l}\text { STD-inpatient } \\
\text { Mean } \\
\text { (Min-Max, SD) }\end{array}$ & $P$-value & $\begin{array}{l}\text { Spearman } \\
\text { correlation } \\
\text { coefficient }\end{array}$ & $P$-value & $\begin{array}{l}\text { Spearman } \\
\text { correlation } \\
\text { coefficient }\end{array}$ & $P$-value \\
\hline Patient overall care experience & $97(75-100,30)$ & $88(24-100,16)$ & $<0.001$ & & & & \\
\hline Satisfaction with preparation & $94(60-100,10)$ & $93(53-100,12)$ & 0.356 & .453 & 0.001 & 0.238 & 0.108 \\
\hline Satisfaction with hospital stay & $96(50-100,9)$ & $85(0-100,23)$ & $<0.001$ & .614 & $<0.001$ & .450 & 0.001 \\
\hline Satisfaction with home care & $89(11-100,20)$ & $92(39-100,13)$ & 0.678 & .661 & $<0.001$ & .411 & 0.004 \\
\hline Satisfaction with pain management & $93(18-100,14)$ & $87(0-100,21)$ & 0.002 & .582 & $<0.001$ & .357 & 0.014 \\
\hline Satisfaction with functional recovery & $95(70-100,7)$ & $84(1-100,20)$ & $<0.001$ & .486 & 0.001 & .374 & 0.01 \\
\hline Satisfaction with wound management & $95(18-100,13)$ & $85(0-100,18)$ & $<0.001$ & .614 & $<0.001$ & 0.124 & 0.405 \\
\hline Satisfaction with overall result & $96(70-100,8)$ & $92(0-100,17)$ & 0.187 & .578 & $<0.001$ & .420 & 0.003 \\
\hline
\end{tabular}

statistically significant for pain management, postoperative recovery, hospital stay, home care and overall results $(p<0.014)$.

\section{Discussion}

The qualitative analysis demonstrated that the quality of support received by the patients throughout the episode of care is important to their THA/TKA experience, and that overall, patients had a better experience with the ERAS-outpatient program because they felt better supported by staff dedicated to ambulatory surgery in the ward, they experienced less postoperative inconvenience, went home sooner, and recovered more quickly. Furthermore, patients suggested that their THA/TKA experience could possibly be enhanced by improving the information given in the preparation phase, providing more postoperative physiotherapy sessions at home if needed and ensuring better coherence of care between hospital and home care teams. The bivariate analyzes revealed a weak to moderate positive correlations between patient experience and satisfaction with pain management, postoperative recovery, hospital stay, home care and overall results. Taken separately, these findings enhance our comprehension of the patient THA/TKA experience. Nevertheless, this study's overarching objective was to gain a more in-depth understanding of the patient experience for both STD-inpatient and ERAS-outpatient THA/TKA by combining the strengths of quantitative and qualitative approaches [20]. Consequently, qualitative and quantitative results were integrated together and compared to the literature in the following paragraphs.

\section{Patients characteristics}

Bivariate analyses did not find relationship between patients' characteristics and their overall care experience nor was it raised by the qualitative analysis. Taken together, these findings suggest that patients' characteristics are not associated with their overall care experience and that the enhanced patient experience in the ERASoutpatient programs was unlikely to be associated with the difference in patients' age and comorbidities between surgeries.

\section{Clinical outcomes}

In this cohort of patients, it was shown that compared to the STD-inpatient, the ERAS-outpatient program resulted in fewer complications, similar postoperative pain with less opioid consumption and sooner functional recovery [12]. These findings were corroborated by the present qualitative analysis as patients clearly discussed how clinical outcomes, such as adverse events, pain, and functional recovery, affected their experience. However similarly to other studies, no significant statistical correlation between clinical outcomes and the patient experience was found [23-25]. These divergent quantitative and qualitative findings underscored the importance of going beyond quantitative assessment alone to truly understand what influences and how it affects the patients' care experience.

\section{Postoperative recovery}

Sooner and better recovery after surgery is the main goal of ERAS interventions [26]. The resulting optimized recovery was a major reason why patients reported a better THA/TKA experience with ERAS-outpatient. The ERAS-outpatient epidural-sedation combination had the advantage over spinal anesthesia of eliminating the distress associated with being awake during the operation and contributed to make patients feel better and recover 
more quickly after surgery $[27,28]$. The earlier ambulation after surgery contributed to reduce complications and to improve pain, muscles strength, range of motion, gait and overall function in the post-acute phase which may explain why patients enjoyed their ERAS-outpatient recovery, even though the exercise program was similar $[16,29-31]$. The earlier ambulation also helped reduce the number of rehabilitation sessions needed to reach functional autonomy which was greatly appreciated by the patients $[12,29]$. Thus, by improving the recovery process, the ERAS-outpatient program optimized the overall patients' experience. Inversely, Johansson Stark et al. [32] observed that a positive patient experience increased the likelihood of better postoperative recovery. Consequently, as recommended by Doyle et al. [11], all three pillars of quality of care (patient safety, clinical effectiveness and patient experience) should be viewed as a group and interventions should aim to improve all three to provide high-quality care in THA/TKA programs.

\section{Pain management}

Pain management is crucial after THA/TKA, as unrelieved pain can negatively affect recovery and patient experience [33-37]. With outpatient programs, patients become quickly responsible for their pain management and it was shown to often result in unrelieved pain [34, 38]. When self-medicating, patients are often reluctant to take the prescribed analgesics because they are not sure when to take them or because they are afraid of developing an addiction [34, 35, 38]. This highlights the importance of educating patients so that they can be confident in the postoperative process and demonstrate the necessary coping skills [39]. Sharing advice from former patients could further empower patients in the process of care, thereby increasing their confidence in managing their pain appropriately [40-42]. The pre-discharge education provided in the ERAS-outpatient program was key to make participants appreciated being able to selfmanage their consumption of opioid-sparing analgesics, as this allowed them to relieve their pain quickly, without having to wait for nursing staff and with less fear of developing a dependency. This method of self-medication, when applied appropriately, has been shown to be strongly associated with better overall pain relief, less need for additional analgesia and, therefore, a better care experience [43].

\section{Hospital stay and home care}

The quality of hospital and home care is important to the overall patients' experience and their satisfaction with these components of care is positively associated to it. The patients appreciated care providers who were competent, engaged and interested in helping them recover, and recognized the added value of being cared by nurses and physiotherapists specialized in the ERAS-outpatient program [34]. However, a care provider deemed unprofessional or not cohesive with the medical team instructions increase patients' uncertainty [34]. Fortunately, patients benefited from having a nurse and a physiotherapist, from the hospital team, available by phone to answer doubts, ease concerns, and provide information which helped to improve the overall provider-patient relationship [44, 45]. Additionally after ERAS-outpatient, a caregiver was present at home in the early postoperative days, to help alleviate the uncertainties of home care and temper the additional concerns of returning home quickly $[35,46]$. The present findings reinforced those of McMurray et al. [45] stating that relationships between patients and care providers are a key component of the patient experience and that aspects such as caring, empathy, respect and perceived provider expertise all influenced the overall experience [45]. On the other hand, the poor home care experience by some patients underscores the importance of improving and standardizing the quality of home care and educating home care providers on the rehabilitation protocol in order to provide a consistent message that will improve patient experience and also potentially postoperative outcomes [47].

\section{Overall results}

Satisfaction with THA/TKA overall results is positively correlated with patient overall care experience. However, patients did not express a difference between their ERASoutpatient and STD-inpatient surgery overall results, which can be explained by the similar PROMs at the last follow-up [12]. Indeed, Black et al. [48] observed a weak positive correlation between patient-reported experience measures (PREMs) and patient-reported outcome measures (PROMs) at 6 months after a THA/TKA. Thus, while it is unlikely that satisfaction with the THA/TKA overall results contributed much to the better patient experience with the ERAS-outpatient program found in this cohort, they are still significantly related.

\section{Study limitations}

The present results must be interpreted taking into consideration its limitations. First, while adequate, our sample size was relatively small for bivariate analyses, and they did not provide information regarding causality or direction. Nevertheless, this limitation was mostly overcome by the mixed methods design which enable to obtain a complete understanding of the patient experience and its associated factors by integrating qualitative data to the quantitative data. Second, unlike the case with interviews, our survey-based instrument did not allow us to ask sub-questions that 
may have allowed to further deepen the participants' points of view. However, the large sample size by qualitative research standards was more than sufficient to gather a great variety of perspectives and experiences. Third, all surgeries were performed at the same Canadian tertiary centre and patients eligible for ERASoutpatient had to meet specific inclusion criteria, resulting in a relatively young and healthy cohort which may limit the generalizability of the present findings to other settings or populations. Fourth, all STD-inpatient surgeries were performed before the ERAS-outpatient ones for which patients were older and more comorbid. Nevertheless, the comparison of patient satisfaction between the first and the second procedure in bilateral asynchronous TKA showed no difference and patients being older would had negatively impacted the ERASoutpatient outcomes [49]. Fifth, participants had to recall their surgeries to answer the Patient Experience questionnaire. Yet, many strategies were put in place to minimize the impact of patient recall: participants were their own comparison, they were blind to the study hypothesis, they could take as much time as they needed to answer, data collection was standardized and done simultaneously for both programs [50].

\section{Conclusion}

This study demonstrated that support throughout the care episode is key to the patient experience and that by reducing postoperative inconvenience, offering enhanced pain management and dedicated support in the ward, enabling to go home sooner and to recover and regain function sooner, ERAS-outpatient arthroplasty programs resulted in an optimized patient experience compared to STD-inpatient practice. Furthermore, patients' experience of THA/TKA could benefit from optimizing the information provided, the rehabilitation program and the coherence between care teams.

\section{Abbreviations}

ERAS: Enhanced Recovery after Surgery; PREMs: Patient reported experience measures; PROMs: Patient Reported Outcome Measurement Study; NRS: Numeric rating scale; THA: Total hip arthroplasty; THA/TKA: Total hip and knee arthroplasty; TKA: Total knee arthroplasty; STD: Standard.

\section{Supplementary Information}

The online version contains supplementary material available at https://doi. org/10.1186/s12891-021-04847-9.

Additional file 1.

\section{Acknowledgements}

We thank Serge Marquis and Margaux Courgeon for helping with the medical records assessment. A. Hardy thanks the Department of Biomedical Sciences of the Université de Montréal for the excellence scholarship received while conducting this study.

\section{Authors' contributions}

A. Hardy as the first author was involved in conceptualization, data curation, formal analysis, investigation, methodology, project administration, resources and software management, validation, visualization and writing the original draft. J.H.G. contributed to conceptualization, data curation, formal analysis, investigation and validation. A.H., K.P. \& F.D. helped with conceptualization, methodology, supervision, and validation. P-A.V. as senior author contributed to conceptualization, formal analysis, investigation, methodology, project administration, resources, supervision, validation and writing the original draft. All authors contributed to final editing and review of the manuscript. All authors have read and approved the final version submitted.

\section{Funding}

This research received unrestricted funding from the Maisonneuve-Rosemont Hospital Foundation.

\section{Availability of data and materials}

The datasets used and/or analysed during the current study are available from the corresponding author on reasonable request.

\section{Declarations}

\section{Ethics approval and consent to participate}

The ethical approval for this study was obtained from the Comité d'éthique de la recherche du CIUSSS de I'Est-de-I'Ille-de-Montréal \#2021-2420. The informed written consent was obtained for all participants approved by Comité d'éthique de la recherche du CIUSSS de l'Est-de-l'Île-de-Montréal. The study was conducted in accordance with the Declaration of Helsinki.

\section{Consent for publication \\ Not applicable.}

\section{Competing interests}

P-A. V. reports royalties from Microport, grants, personal fees and others from Stryker, grants, personal fees and others from Johnson and Johnson, and grants from Zimmer; all outside the submitted work. All authors declare that they have no competing interests.

\section{Author details}

'Department of Biomedical Sciences, Faculty of Graduate and Postdoctoral Studies, Université de Montréal, Montreal, Quebec, Canada. ${ }^{2}$ Hôpital Maisonneuve-Rosemont, Surgery Department, Université de Montréal, Montreal, Quebec, Canada. ${ }^{3}$ Centre de recherche de l'Hôpital MaisonneuveRosemont, Quebec, Montreal, Canada. ${ }^{4}$ School of Rehabilitation, Faculty of Medicine, Université de Montréal, Montreal, Quebec, Canada. ${ }^{5}$ Centre de recherche interdisciplinaire en réadaptation du Montréal métropolitain (CRIR), Montreal, Quebec, Canada. ${ }^{6}$ Centre de recherche en éthique (CRÉ), Université de Montréal, Montreal, Quebec, Canada. 'Department of Rehabilitation, Faculty of Medicine, Université Laval, Quebec, Canada. ${ }^{8}$ Center for Interdisciplinary Research in Rehabilitation and Social Integration (Cirris), Quebec, Canada. ${ }^{9}$ Personalized Arthroplasty Society, Atlanta, Georgia, USA. ${ }^{10}$ Duval Orthopaedic Clinic, Laval, Quebec, Canada.

Received: 3 June 2021 Accepted: 5 November 2021

Published online: 23 November 2021

References

1. Bombardier C, Hawker G, Mosher D. The Impact of Arthritis in Canada: Today and Over the Next 30 Years. 2011. https://www.arthritisalliance.ca/ images/PDF/eng/Initiatives/20111022_2200_impact_of_arthritis.pdf.

2. Kort NP, Barrena EG, Bédard M, et al. Resuming elective hip and knee arthroplasty after the first phase of the SARS-CoV-2 pandemic: the European hip society and European knee associates recommendations. Knee Surg Sports Traumatol Arthrosc. 2020;28(9):2730-46. https://doi.org/10. 1007/s00167-020-06233-9. 
3. Wilson JM, Schwartz AM, Farley KX, Roberson JR, Bradbury TL, Guild GN. Quantifying the backlog of Total hip and knee arthroplasty cases: predicting the impact of COVID-19. HSS J. 2020;4:1-7. https://doi.org/10.1007/ s11420-020-09806-Z.

4. Zhu S, Qian W, Jiang C, Ye C, Chen X. Enhanced recovery after surgery for hip and knee arthroplasty: a systematic review and meta-analysis. Postgrad Med J. 2017;93(1106):736-42. https://doi.org/10.1136/postgradme dj-2017-134991.

5. Büttner M, Mayer AM, Büchler B, Betz U, Drees P, Susanne S. Economic analyses of fast-track total hip and knee arthroplasty: a systematic review. Eur J Orthop Surg Traumatol. 2020;30(1):67-74. https://doi.org/10.1007/ s00590-019-02540-1.

6. Malviya A, Martin K, Harper I, et al. Enhanced recovery program for hip and knee replacement reduces death rate. Acta Orthop. 2011;82(5):57781. https://doi.org/10.3109/17453674.2011.618911.

7. Kehlet $\mathrm{H}$, Slim K. The future of fast-track surgery. BJS Br J Surg. 2012;99(8):1025-6. https://doi.org/10.1002/bjs.8832.

8. Vehmeijer SBW, Husted H, Kehlet H. Outpatient total hip and knee arthroplasty. Acta Orthop. 2018;89(2):141-4. https://doi.org/10.1080/17453674. 2017.1410958.

9. Kehlet $\mathrm{H}$. Enhanced recovery after surgery (ERAS): good for now, but what about the future? Can J Anesth Can Anesth. 2015;62(2):99-104. https://doi.org/10.1007/s12630-014-0261-3.

10. Darzi L. High Quality Care For All. 2008. https://webarchive.nationalar chives.gov.uk/20130105053023/, http://www.dh.gov.uk/en/Publicatio nsandstatistics/Publications/PublicationsPolicyAndGuidance/DH_ 085825.

11. Doyle C, Lennox L, Bell D. A systematic review of evidence on the links between patient experience and clinical safety and effectiveness. BMJ Open. 2013;3(1):e001570. https://doi.org/10.1136/bmjopen-2012-001570.

12. Hardy A, Courgeon M, Pellei K, Desmeules F, Loubert C, Vendittoli P-A. Improved Clinical Outcomes of Outpatient Enhanced Recovery Hip and Knee Replacements in Comparison to Standard Inpatient Procedures: A Study of Patients Who Experienced Both. Orthop Traumatol Surg Res. Accepted for publication OTSR-D-21-00496, 2021. Preprint online publication. https://doi.org/10.21203/rs.3.rs-418395/v1.

13. Vendittoli P-A, Pellei K, Desmeules F, et al. Enhanced recovery shortstay hip and knee joint replacement program improves patients outcomes while reducing hospital costs. Orthop Traumatol Surg Res. 2019;105(7):1237-43. https://doi.org/10.1016/j.otsr.2019.08.013.

14. Goyal N, Chen AF, Padgett SE, et al. Otto Aufranc award: a multicenter, randomized study of outpatient versus inpatient Total hip arthroplasty. Clin Orthop. 2017;475(2):364-72. https://doi.org/10.1007/ s11999-016-4915-z.

15. Deng Q-F, Gu H-Y, Peng W, et al. Impact of enhanced recovery after surgery on postoperative recovery after joint arthroplasty: results from a systematic review and meta-analysis. Postgrad Med J. 2018;94(1118):67893. https://doi.org/10.1136/postgradmedj-2018-136166.

16. den Hertog A, Gliesche K, Timm J, Mühlbauer B, Zebrowski S. Pathwaycontrolled fast-track rehabilitation after total knee arthroplasty: a randomized prospective clinical study evaluating the recovery pattern, drug consumption, and length of stay. Arch Orthop Trauma Surg. 2012;132(8):1153-63. https://doi.org/10.1007/s00402-012-1528-1.

17. Berg U, W-Dahl A, Rolfson O, Nauclér E, Sundberg M, Nilsdotter A. Influence of fast-track programs on patient-reported outcomes in total hip and knee replacement (THR/TKR) at Swedish hospitals 2011-2015: an observational study including 51,169 THR and 8,393 TKR operations. Acta Orthop. 2020;91(3):306-12. https://doi.org/10.1080/17453674.2020.17333 75.

18. Patient Experience 101 - Why? - The Beryl Institute - Improving the Patient Experience. Accessed Mar 24, 2021. https://www.theberylinstitu te.org/page/PX101_Why

19. Wolf JA, Niederhauser V, Marshburn D, LaVela SL. Defining Patient Experience. Patient Exp J. 2014;1 (1):7-19. https://doi.org/10.35680/2372-0247. 1004.

20. Creswell JW. Research Design: Qualitative, Quantitative, and Mixed Methods Approaches. 4th ed. Thousand Oaks: SAGE Publications; 2014.

21. Braun V, Clarke V. Reflecting on reflexive thematic analysis. Qual Res Sport Exerc Health. 2019;11(4):589-97. https://doi.org/10.1080/2159676X.2019. 1628806 .
22. Creswell JW, Plano Clark VL. Designing and Conducting Mixed Methods Research. 3rd ed. Thousand Oaks: SAGE Publications; 2017.

23. Anhang Price R, Elliott MN, Zaslavsky AM, et al. Examining the role of patient experience surveys in measuring health care quality. Med Care Res Rev. 2014;71(5):522-54. https://doi.org/10.1177/1077558714541480.

24. Bovonratwet P, Shen TS, Islam W, Sculco PK, Padgett DE, Su EP. Is there an association between negative patient-experience comments and perioperative outcomes after primary Total hip arthroplasty? J Arthroplasty Published online January. 2021;20. https://doi.org/10.1016/j.arth.2021.01. 023.

25. LaVela SL, Gallan AS. Evaluation and measurement of patient experience. Patient Exp J. 2014;1 (1):28-36. https://doi.org/10.35680/2372-0247.1003.

26. Kehlet $\mathrm{H}$, Wilmore DW. Evidence-based surgical care and the evolution of fast-track surgery. Ann Surg. 2008;248(2):189-98. https://doi.org/10.1097/ SLA.0b013e31817f2c1a.

27. Karlsson AC, Ekebergh M, Mauléon AL, Österberg SA. "Is that my leg?" patients' experiences of being awake during regional anesthesia and surgery. J Perianesthesia Nurs Off J Am Soc Perianesthesia Nurses. 2012;27(3):155-64. https://doi.org/10.1016/j.jopan.2012.02.005.

28. Bergman $M$, Stenudd $M$, Engström $\AA$. The experience of being awake during orthopaedic surgery under regional anaesthesia. Int J Orthop Trauma Nurs. 2012;16(2):88-96. https://doi.org/10.1016/j.ijotn.2011.08.004.

29. Labraca NS, Castro-Sánchez AM, Matarán-Peñarrocha GA, Arroyo-Morales M, Sánchez-Joya M. del M, Moreno-Lorenzo C. benefits of starting rehabilitation within 24 hours of primary total knee arthroplasty: randomized clinical trial. Clin Rehabil. 2011;25(6):557-66. https://doi.org/10.1177/ 0269215510393759.

30. Henderson KG, Wallis JA, Snowdon DA. Active physiotherapy interventions following total knee arthroplasty in the hospital and inpatient rehabilitation settings: a systematic review and meta-analysis. Physiotherapy. 2018;104(1):25-35. https://doi.org/10.1016/.j.physio.2017.01.002.

31. Lei Y-T, Xie J-W, Huang Q, Huang W, Pei F-X. Benefits of early ambulation within $24 \mathrm{~h}$ after total knee arthroplasty: a multicenter retrospective cohort study in China. Mil Med Res. 2021;8(1):17. https://doi.org/10.1186/ s40779-021-00310-x.

32. Stark $\AA \mathrm{J}$, Charalambous $\mathrm{A}$, Istomina $\mathrm{N}$, et al. The quality of recovery on discharge from hospital, a comparison between patients undergoing hip and knee replacement - a European study. J Clin Nurs. 2016;25(1718):2489-501. https://doi.org/10.1111/jocn.13278.

33. Lo LWT, Suh J, Chen JY, et al. Early Postoperative Pain After Total Knee Arthroplasty Is Associated With Subsequent Poorer Functional Outcomes and Lower Satisfaction. J Arthroplasty. 2021;25. https://doi.org/10.1016/j. arth.2021.02.044.

34. Specht K, Kjaersgaard-Andersen P, Pedersen BD. Patient experience in fast-track hip and knee arthroplasty - a qualitative study. J Clin Nurs. 2016;25(5-6):836-45. https://doi.org/10.1111/jocn.13121.

35. Specht K, Agerskov H, Kjaersgaard-Andersen P, Jester R, Pedersen BD. Patients' experiences during the first 12 weeks after discharge in fast-track hip and knee arthroplasty - a qualitative study. Int J Orthop Trauma Nurs. 2018;31:13-9. https://doi.org/10.1016/j.ijotn.2018.08.002.

36. Berg U, Berg M, Rolfson O, Erichsen-Andersson A. Fast-track program of elective joint replacement in hip and knee-patients' experiences of the clinical pathway and care process. J Orthop Surg. 2019;14. https://doi. org/10.1186/s13018-019-1232-8.

37. Lane JV, Hamilton DF, MacDonald DJ, Ellis C, Howie CR. Factors that shape the patient's hospital experience and satisfaction with lower limb arthroplasty: an exploratory thematic analysis. BMJ Open. 2016;6(5):e010871. https://doi.org/10.1136/bmjopen-2015-010871.

38. Churchill L, Pollock M, Lebedeva Y, et al. Optimizing outpatient total hip arthroplasty: perspectives of key stakeholders. Can J Surg. 2018;61 (6):370-6. https://doi.org/10.1503/cjs.016117.

39. Høvik LH, Aglen B, Husby VS. Patient experience with early discharge after total knee arthroplasty: a focus group study. Scand J Caring Sci. 2018;32(2):833-42. https://doi.org/10.1111/scs.12514.

40. Garzón-Rey JM, Arza-Valdés A, Nuevo-Gayoso M, Aguiló J. Effectiveness of patient empowerment over stress related to knee arthroplasty surgery. Enferm Clínica Engl Ed. 2018;28(3):186-93. https://doi.org/10.1016/j. enfcle.2018.05.001.

41. Jones S, Alnaib M, Kokkinakis M, Wilkinson M. St Clair Gibson a, Kader D. pre-operative patient education reduces length of stay after knee joint 
arthroplasty. Ann R Coll Surg Engl. 2011;93(1):71-5. https://doi.org/10. 1308/003588410X12771863936765.

42. Pellino T, Tluczek A, Collins M, et al. Increasing self-efficacy through empowerment: preoperative education for orthopaedic patients. Orthop Nurs. 1998;17(4):48-51 54-59.

43. Moore R, Derry S, Straube S, Ireson-Paine J, Wiffen P. Validating speed of onset as a key component of good analgesic response in acute pain. Eur J Pain Lond Engl. 2015;19(2):187-92. https://doi.org/10.1002/ejp.536.

44. Strickland LH, Kelly L, Hamilton TW, Murray DW, Pandit HG, Jenkinson C. Early recovery following lower limb arthroplasty: qualitative interviews with patients undergoing elective hip and knee replacement surgery. Initial phase in the development of a patient-reported outcome measure. J Clin Nurs. 2018;27(13-14):2598-608. https://doi.org/10.1111/jocn.14086.

45. McMurray J, McNeil H, Lafortune C, Black S, Prorok J, Stolee P. Measuring patients' experience of rehabilitation services across the care continuum. Part II: key dimensions. Arch Phys Med Rehabil. 2016;97(1):121-30. https://doi.org/10.1016/j.apmr.2015.08.408.

46. Sjøveian AKH, Leegaard M. Hip and knee arthroplasty - patient's experiences of pain and rehabilitation after discharge from hospital. Int J Orthop Trauma Nurs. 2017;27:28-35. https://doi.org/10.1016/j.ijotn.2017. 07.001.
47. Dibbelt S, Schaidhammer M, Fleischer C, Greitemann B. Patient-doctor interaction in rehabilitation: the relationship between perceived interaction quality and long-term treatment results. Patient Educ Couns. 2009;76(3):328-35. https://doi.org/10.1016/j.pec.2009.07.031.

48. Black N, Varaganum M, Hutchings A. Relationship between patient reported experience (PREMs) and patient reported outcomes (PROMs) in elective surgery. BMJ Qual Saf. 2014;23(7):534-42. https://doi.org/10. 1136/bmjqs-2013-002707.

49. Clement ND, Merrie KL, Weir DJ, Holland JP, Deehan DJ. Asynchronous bilateral Total knee arthroplasty: predictors of the functional outcome and patient satisfaction for the second knee replacement. J Arthroplast. 2019;34(12):2950-6. https://doi.org/10.1016/j.arth.2019.06.056.

50. Hassan E. Recall Bias can be a Threat to Retrospective and Prospective Research Designs. Internet J Epidemiol. 2005;3(2) Accessed May 12, 2020 http://ispub.com/IJE/3/2/13060.

\section{Publisher's Note}

Springer Nature remains neutral with regard to jurisdictional claims in published maps and institutional affiliations.
Ready to submit your research? Choose BMC and benefit from:

- fast, convenient online submission

- thorough peer review by experienced researchers in your field

- rapid publication on acceptance

- support for research data, including large and complex data types

- gold Open Access which fosters wider collaboration and increased citations

- maximum visibility for your research: over $100 \mathrm{M}$ website views per year

At BMC, research is always in progress.

Learn more biomedcentral.com/submissions 